\title{
Prior fixed ratio training and durable persistence in rats
}

\author{
PAUL T. P. WONG \\ Trent University, Peterboro, Ontario, Canada \\ and \\ ABRAM AMSEL \\ University of Texas at Austin, Austin, Texas 78712
}

\begin{abstract}
Following barpress training with different terminal fixed ratios (FR), rats were given the interpolated experiences of runway acquisition and extinction (as part of another experiment) followed by 2 months of vacation. Then they were tested in FR 10 barpress reacquisition, FR 10 barpress extinction, consistently reinforced runway reacquisition, and a second runway extinction. In a start (response initiation) measure, resistance to extinction during the FR 10 extinction and in the second runway extinction was positively related to the terminal FR values of the initial barpress training, an indication of highly durable differential persistence effects attributable to the initial training to different terminal fixed ratios of barpress responding.
\end{abstract}

It has been observed that the widely held notion about the permanence of habits lacks empirical support (Gleitman, 1971). Near-perfect retention of long-term memories in animals is the exception (e.g., Gleitman \& Jung, 1963; Maier \& Gleitman, 1967), while some forgetting is the rule (e.g., Honig \& James, 1971).

One may argue, however, that retention is only one aspect of the durability of learned associations; persistence must also be considered an important indicant of the permanence of habits. Retention reflects protection from interference or decay, and is typically inferred from the degree of performance decrement following a retention interval that necessarily involves some interpolated activity. Persistence, on the other hand, reflects protection from extinctive suppression, and is typically inferred from pe:-formance decrement during or following a treatment designed to suppress expression of the habit (e.g., extinction or punishment). The possible interrelations between retention and persistence have not been explored, but there is some indirect evidence of independence between these two dimensions of habit. For example, a greater number of training trials invariably leads to greater retention (e.g., Ebbinghaus, reprinted 1964), but may decrease persistence as mea-

The experiment was supported by Grant GB-14990X from the National Science Foundation to Abram Amsel. Preparation of manuscript was supported both by GB-14490X and by Grant 3 A8635 from the National Research Council to P. T. P. Wong. Requests for reprints should be sent to Abram Amsel, Department of Psychology, University of Texas at Austin, Austin, Texas 78712 . sured by resistance to extinction (e.g., Ison, 1962; Traupmann, 1972). It would seem that we cannot arrive at a complete understanding of the permanence of habits without considering both retention and persistence.

Our research on durable persistence has typically employed a four-phase paradigm: differential treatment (D), extinction (E), nondifferential treatment $(\mathrm{N})$, and a second extinction ( $\left.E^{\prime}\right)$. The rationale for this D-E-N-E' paradigm is as follows: During Phase 1 , subjects are exposed to different treatments designed to induce differential persistence. Phase 2 reveals this differential persistence in terms of different rates of extinction, thus confirming the effectiveness of the Phase 1 treatment; however, Phase 2 may not provide a pure measure of differential persistence because it may be confounded by differential discriminability between Phase 1 and Phase 2. In Phase 3, subjects are exposed to nondifferential experiences or treatments. Phase 4, in which differential persistence can be attributed only to the differential treatment in Phase 1 because of systematically equivalent treatments in Phases 2 and 3, provides evidence of the durability of treatment effects on persistence. This basic paradigm may be modified in several ways. For example, Phase 1 and Phase 3 may involve the same or different response systems, and retention intervals of various durations or different kinds of interpolated activities may be included prior to the Phase 4 persistence test. Employing the D-E-N-E' paradigm, we have found that persistence resulting from a partial-delayof-reinforcement treatment is not durable in that it does not survive Phases 2 and 3 (Wong, Traupmann, \& Brake, 1974), but that persistence resulting from 
the partial-reinforcement treatment is extremely durable (e.g., Amsel, Wong, \& Traupmann, 1971).

The present experiment extends the phenomenon of durable persistence in two important ways. First, the degree of durable persistence was related to a treatment involving different fixed ratio (FR) requirements in barpress training. Secondly, as a departure from the basic D-E-N-E' paradigm, the durable nature of persistence arising from the differential FR requirements was measured both in a lever box and in a runway. That resistance to extinction is positively related to terminal FR requirements is a longestablished discovery (Boren, 1961). Recently, we demonstrated in a transfer paradigm a positive relation between degree of persistence in a runwayextinction test and terminal FR values in prior barpress training (McCuller, Wong, \& Amsel, 1976). ${ }^{1}$ The present experiment is a long-term follow-up of the subjects from the McCuller et al. experiment to determine whether the persistence levels generated by the different FR requirements in the original barpress training would survive (a) runway acquisition and extinction, (b) 2 months of vacation from testing, and (c) FR 10 reacquisition and continuously reinforced runway reacquisition and still be reflected in final barpress and runway extinction tests.

\section{METHOD}

\section{Subjects}

The subjects were 44 male Holtzmann rats previously given barpress training with different FR requirements and runway acquisition and extinction in Experiment II of McCuller, Wong, and Amsel.(1976). They were maintained on $12 \mathrm{~g}$ of Wayne Lab Chow per day with ad-lib water.

\section{Apparatus \\ The straight runway and barpress chambers were those described in McCuller et al. Briefly, the runway measured $190 \mathrm{~cm}$ long, $7.6 \mathrm{~cm}$ wide, and $10.2 \mathrm{~cm}$ high, and, in connection with photoelectric circuitry, provided five time measures: start, Run 1 , Run 2, Run 3, and goal. The three barpress chambers were housed in sound-attenuated boxes, mounted with retractable bars, and interfaced with a Grason-Stadler SCAT system which was respon- sible for all controlling and recording functions.}

\section{Procedure}

All four groups (11 subjects per group) received 50 days of barpress training, four trials per day. The first trial was initiated by the pressing of a start button by the experimenter as soon as the subject was placed in the barpress chamber and the soundattenuated box was closed. Subsequent trials were preceded by a $60-\mathrm{sec}$ time-out. All trials began with the extension of the operant lever and ended with the retraction of the lever which occurred after completion of the FR requirement or the elapse of $30 \mathrm{~min}$, whichever came first. Each completion of a required FR produced a 300-mg Noyes pellet.

Group FR 10 remained on FR 10 throughout the 50 days of training. For the other three groups, the first 6 days were on FR 10, but thereafter the ratio requirement was increased by 10 after each 4 days of training until the appropriate terminal ratio requirement (FR 40, FR 80, or FR 120) was reached. These terminal FR values remained unchanged for the remainder of barpress training.
During the runway training phase, all subjects received 12 rewarded trials and 32 extinction triais at one trial a day.

The above two phases were completed in the McCuller et al. study and constituted the only differential treatment for the present investigation.

After the completion of the McCuller et al. experiment, all subjects were given 2 months of "vacation" away from any testing (Phase 3). During this period, subjects were handled only to be weighed. In the first month, subjects were maintained on about $20 \mathrm{~g}$ per day. In the second month, they were again reduced to a regimen of $12 \mathrm{~g}$ a day.

In Phase 4, all subjects were given 8 days of reinforced FR 10 barpress training, 4 trials a day, followed by 9 days of FR 10 barpress extinction, at 4 trials a day. In extinction, the 10th response resulted in a click of the food dispenser, but no food pellet, followed by a 60 -sec time-out.

In Phase 5, all subjects received 12 rewarded runway trials (with a $300-\mathrm{mg}$ pellet as reward) and 32 extinction trials, at one trial a day.

\section{RESULTS AND DISCUSSION}

\section{FR Acquisition}

The four groups did not differ during the first 6 days of FR 10 training. Data for the remainder of FR training are shown in Figure 1, which is a much more detailed presentation of the McCuller et al. acquisition data than was provided in the earlier report. The upper panel presents the mean speeds $(1 / \mathrm{sec})$ to initiate the first response following the extension of the lever. The lower panel presents the mean speeds $(10 / \mathrm{sec})$ to complete the first $10 \mathrm{bar}$ presses. The graph shows how each increase in the ratio requirement systematically depressed performance in both the 0-1 and 1-10 measures as compared to groups for which ratio requirements remained unchanged at the same stage of training. Analysis of the data for Days 7-18 revealed a significant Groups by Days interaction $(F=1.81, \mathrm{df}=33 / 440, \mathrm{p}<.01)$ for the 0-1 measure, and a significant Group main effect $(F=7.44, \mathrm{df}=3 / 40, \mathrm{p}<.01)$ for the $1-10$ measure. For Days 19-34, Group main effect was significant for $0-1(F=3.16, \mathrm{df}=3 / 40, \mathrm{p}<.05)$ and $1-10(F=7.44$, df $=3 / 40, p<.01$ ). For Days 35-50, Group main effect was also significant for $0-1(F=3.63)$ and $1-10(F=7.66)$.

The 0-1 data are consistent with the well-established finding that length of the postreinforcement pause increases with FR requirement (Felton \& Lyon, 1966; Ferster \& Skinner, 1957; Powell, 1968). This pause may more accurately be referred to as a preratio pause (Findley, 1962; Griffiths \& Thompson, 1973), especially with respect to pauses occurring before the very first response of the day. Ferster and Skinner (1957, p. 93) commented that long pauses with larger fixed ratios "are clearly not due to factors such as physical exhaustion or fatigue, but to the extremely unfavorable stimuli then present." In a mixed FR schedule, Keehn (1965) observed that longer pauses occurred before and not after the largest ratio. In the present study, preratio pause on the very first trial of 


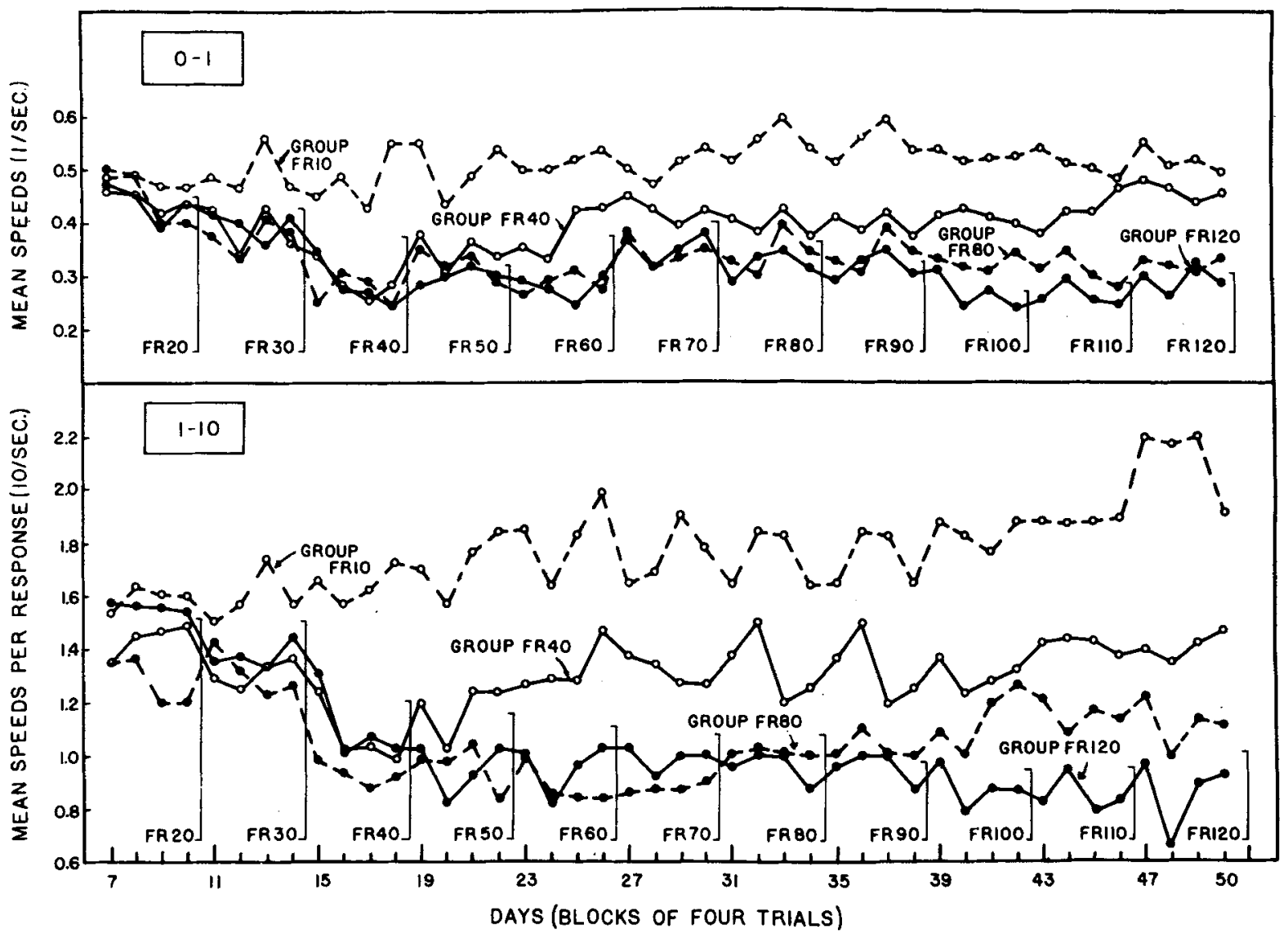

Figure 1. A detailed analysis of speed data during FR barpress training. The vertical brackets indicate the ratio values of grcups included in the brackets.

each day could not possibly be due to fatigue; further, pausing tended to decrease rather than increase on Trials 2 and 3 and showed a slight increase only on Trial 4, although these differences were not significant. Here again, then, is evidence against a fatigue hypothesis of the preratio pause. The alternative hypothesis proposed by Ferster and Skinner (1957) has been supported in several experiments demonstrating the aversive property associated with long ratios (e.g., Azrin, 1961; Cherek \& Pickens, 1970).

According to frustration theory, any increase in the ratio requirement is a potential source of primary frustration $\left(R_{F}\right)$. By definition (Amsel, 1958), such frustration would result from increased delay of reinforcement and decreased percentage or number of reinforcements. Perhaps more important, any increase in the ratio requirement (FR $n+x)$ defines frustrative nonreward because reward previously given after the $\mathrm{n}^{\text {th }}$ response is now withheld. According to this analysis, the higher the ratio, the higher $\mathbf{R}_{\mathrm{F}}$ and consequently the more intense the conditioned anticipatory frustration $\left(\mathrm{r}_{\mathrm{F}}\right)$. Indirect support for this analysis comes from the findings that increasing ratio requirements systematically increases aggressive behavior (e.g., Cherek \& Picken, 1970) and escape behavior (Azrin, 1961; Thompson, 1964, 1965).

Our hypothesis is that prior to the completion of a response chain, anticipatory frustration and its feedback stimulus ( $\left.\mathrm{r}_{\mathrm{F}}-\mathrm{s}_{\mathrm{F}}\right)$ mediates a "try-or-stop" conflict, the approach-avoidance conflict in a straight runway under partial reinforcement conditions (Amsel, 1958) being a special case of this general conflicting tendency. The tendency to try is assumed to increase directly with frequency and magnitude of reinforcement, and inversely with delay of reinforcement; the tendency to stop increases with size of ratio. Therefore, each increment in ratio size increases response latency, as is evident in both the $0-1$ and 1-10 measures. When the ratio requirement is increased indefinitely, the subject will of course reach a breaking point and the large-ratio schedule functions as an extinction operation (cf. Skinner, 1938, p. 282 and p. 293). Another implication is that if the ratio requirement is frozen prior to the breaking point, speed of responding should increase with successive reinforcements at that ratio. Such an incremental trend could be taken to reflect building of persistence, and was evident in Groups FR 10 and FR 40 in the 
0-1 measure, and in all groups except Group FR 120 in the 1-10 measure.

Group differences cannot be attributed to number of reinforcements, because our discrete-trial procedure ensured that all subjects receive one reinforcement per trial. A fatigue interpretation is inadequate in view of these two observations: First, group differences in Trial 1 (where the fatigue factor should be the same for all groups) were of the same magnitude as in the remaining trials, and this could also be inferred from the absence of a significant Groups by Trials interaction. Second, response speeds tended to be an inverted-U function of trials for all groups, with the fastest speeds on Trial 2 or Trial 3.

\section{FR 10 Reacquisition and Extinction ${ }^{2}$}

The results from these phases are shown in Figure 2. During FR 10 reacquisition, all groups performed at a level appropriate for FR 10, and did not differ significantly from each other. In extinction, mean start speeds for Groups FR 120, FR 80, FR 40, and FR 10 were $0.40,0.37,0.33$, and 0.25 , respectively. Although these group differences did not reach the conventional level of significance in analysis of

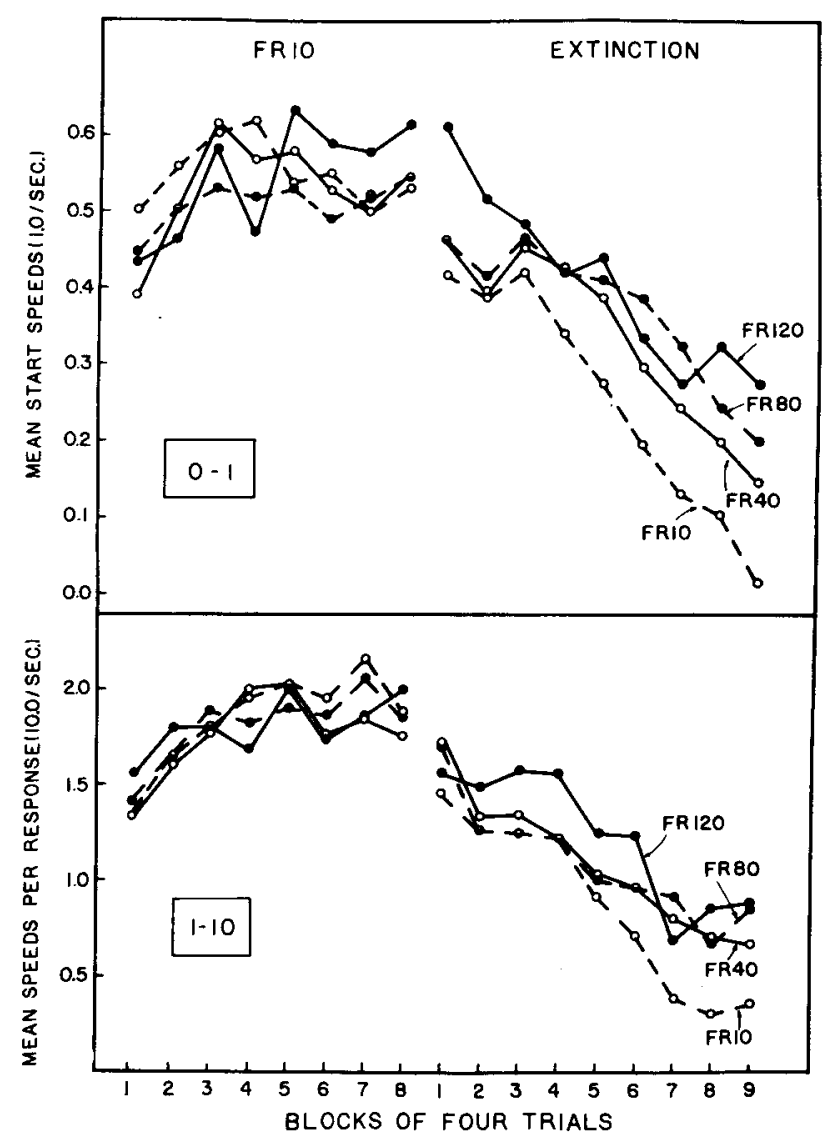

Figure 2. Speed data during FR 10 reacquisition and FR 10 extinction. variance, such a systematic ranking according to terminal FR is statistically significant $(p=1 / 4 !=0.04)$. In the 1-10 measure, neither the group main effect nor the Group by Trial Blocks interaction was significant, although terminal FR values were still in the correct order. Consistent with the McCuller et al. finding, the durable persistence effect was more pronounced in the start measure. This result supports the hypothesis that durable persistence is mediated by the conditioning of continued responding to anticipatory frustration, because the "Try vs. Try" conflict should affect mainly the onset of instrumental responding. Once the response has been initiated, proprioceptive and other feedback would tend to be an important part of the stimulus complex. And because proprioceptive feedback varies according to ratio requirements, the shift from higher ratios to FR 10 should result in stimulus generalization decrement, masking to some extent differential persistence effects in the 1-10 measure.

\section{Runway Reacquisition and the Second Extinction}

There were no significant differences between groups in any of the measures during runway reacquisition; therefore, only terminal reacquisition and extinction data are shown in Figure 3. Just as during barpress extinction, significant differential persistence was shown only in the start measure $(F=3.25$, df $=3 / 40, p<.05)$. In other measures, the ordering of curves was generally from high to low FR values, but no Groups main effect was significant.

These results suggest that graded partial reinforcement effects resulting from different terminal FR values are extremely durable: some effects of this treatment survived runway acquisition and extinction, a 2-month retention-vacation interval, and FR 10 reacquisition, to show up in an FR 10 extinction; and then survived this extinction and a subsequent runway reacquisition to show up in a second runway extinction treatment. It goes almost without saying that this kind of finding poses a problem for a number of theoretical accounts of extinction, most particularly perhaps for a discrimination hypothesis (e.g., Bitterman, Fedderson, \& Tyler, 1953; Nevin, 1973), because the final persistence test in the operant box and the runway was preceded by several phases of identical treatment for all groups. One might also wonder about Skinner's concept of the reflex reserve in relation to these findings (Skinner, 1938), but it is not clearly relevant to the present FR 10 extinction test because the dependent variable here was not total number of responses in extinction, but rather time to the first response, or first 10 responses.

We propose that findings of durable persistence effects in a D-E-N-E' paradigm requires a conceptualization of persistence-at least long-term persistence-as a learned disposition to continue 
Figure 3. Speed data during terminal reacquisition (TRA) and the second extinction in the runway.
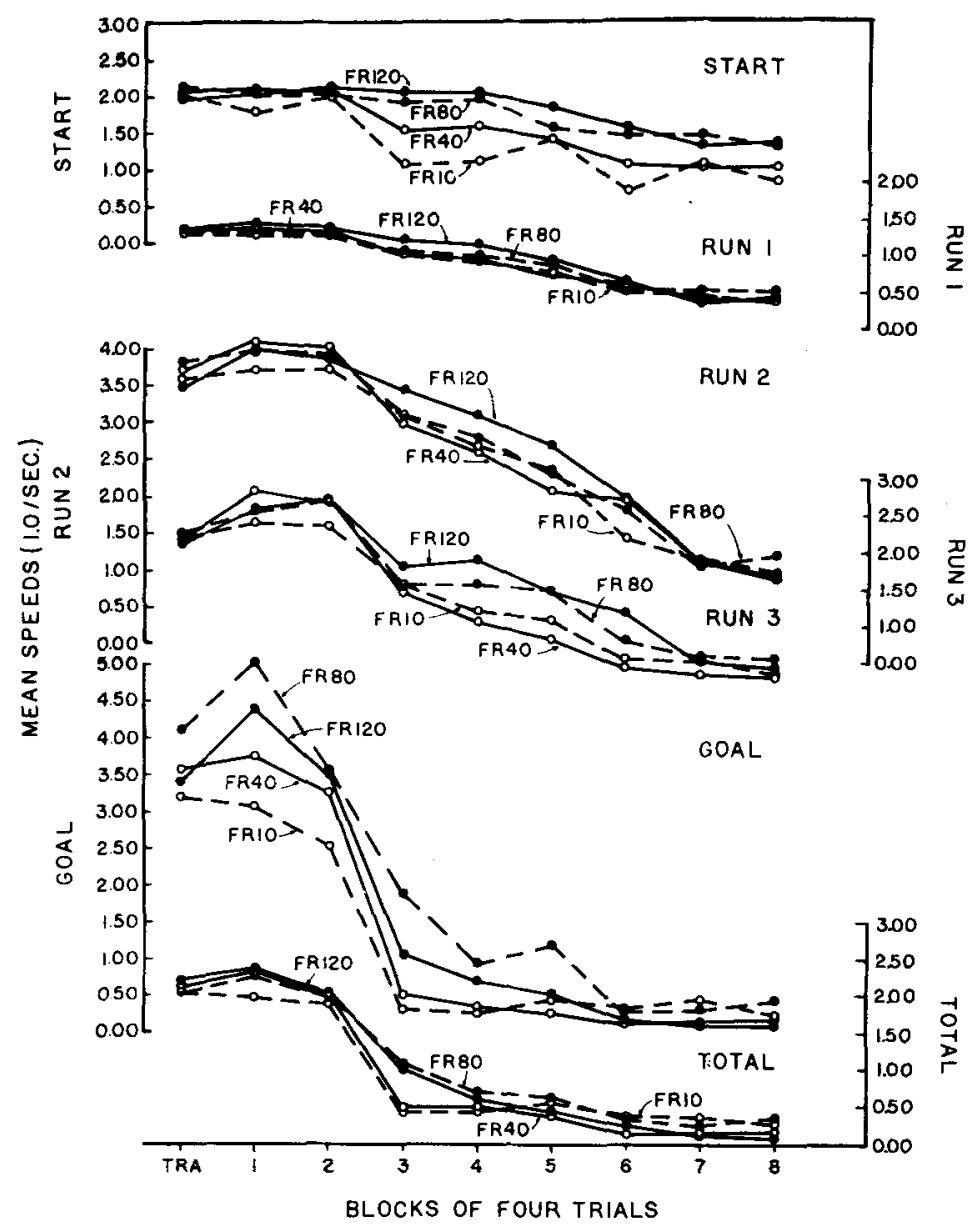

responding in the face of anticipatory frustration. Inasmuch as this disposition becomes a general tendency to cope with frustration, or can so be conceptualized, it may be profitable to think of the mechanism, perhaps a bit loosely, as a highly general response strategy to continue trying. Transsituational and transresponse transfer of persistence, now demonstrated in a number of experiments, has been assumed to be mediated by a mechanism connecting $\mathrm{r}_{\mathrm{F}}-\mathrm{S}_{\mathrm{F}}$ to the initiation of responding (a "Try" mechanism). Such a hypothesis implies that durable persistence effects should be most clearly discernible in measures reflecting response initiation, because the conflict between Try and Try should operate most effectively in the initial portion of an instrumental response chain.

\section{REFERENCES}

Amsel, A., Wong, P. T. P., \& Traupmann, K. L. Short-term and long-term factors in extinction and durable persistence. Journal of Experimental Psychology, 1971, 90, 90-95.

AMSEL, A. The role of frustrative nonreward in noncontinuous reward situations. Psychological Bulletin, 1958, 55, 102-119.

AzRIN, N. H. Time-out from positive reinforcement. Science, 1961 . $133,382-383$.
Bitterman, M. E., Fedderson, W. E., \& Tyler, D. W. Secondary reinforcement and the discrimination hypothesis. American Journal of Psychology, 1953, 66, 456-464.

BOREN, J. J. Resistance to extinction as a function of the fixed ratio. Journal of Experimental Psychology, 1961, 61, 304-308.

Cherex, D. R., \& Pickens, R. Schedule-induced aggression as a function of fixed-ratio value. Joumal of the Experimental Analvsis of Behavior. 1970, 14, 309-312.

Enringhaus, H. Memory: A contribution to experimental psychology. New York: Dover, 1964.

Felton, M., \& Lyon, D. O. The post-reinforcement pause. Journal of the Experimental Analysis of Behavior, 1966, 9. 131-134.

Ferster, C. B., \& Skinner, B. F. Schedules of reinforcement. New York: Appleton-Century-Crofts, 1957.

FindeEY, J. D. An experimental outline for building and exploring multi-operant behavior repertoires. Joumal of the Experimental Analysis of Behavior, 1962, 5, 113-166.

Glertman, H. Forgetting of long-term memories in animals. In W. K. Honig \& P. H. R. James (Eds.), Animal memory. New York: Academic Press, 1971.

Gleitman, H., \& Jung, L. Retention in rats: The effect of proactive interference. Science, 1963, 142, 1683-1684.

Griffiths, R. R., \& Thompson, T. The post-reinforcement pause: A misnomer. Psychological Record, 1973, 23, 229-235.

Honig, W., \& James, P. H. R. Animal memory. New York: Academic Press, 1971.

Ison, J. Experimental extinction as a function of number of reinforcements. Journal of Experimental Psychology, 1962, 64, 314-317. 
KEEHN, J. D. Temporal alternation in the white rat. Journal of the Experimental Analysis of Behavior, 1965, 8, 161-168.

Maier, S. F., \& Gleitman, H. Proactive interference in rats. Psychonomic Science, 1967, 7, 25-26.

McCuller, T., Wong, P. T. P., \& Amsel, A. Transfer of persistence from fixed-ratio barpress training to runway extinction. Animal Learning \& Behavior, 1976, 4, 53-57.

Nevin, J. A. (Ed.) The study of behavior: Learning, motivation, emotion, and instinct. Glenview: Scott, Foresman, 1973.

Powell, R. W. The effect of small sequential changes in fixedratio size upon the post-reinforcement pause. Journal of the Experimental Analysis of Behavior, 1968, 11, 589-593.

SKINNER, B. F. The behavior of organisms: An experimental analysis. New York: Appleton-Century-Crofts, 1938.

Thompson, D. M. Escape from $S D$ associated with fixed-ratio reinforcement. Joumal of the Experimental Analysis of Behavior, $1964,7,1-8$.

Thompson, D. M. Time-out from fixed ratio reinforcement: A systematic replication. Psychonomic Science, 1965, 2, 109-110.

TRAUPMANN, L. Drive, reward, and training parameters, and the overlearning-extinction effect (OEE). Leaming and Motivation, 1972, 3, 359-368.

Wong, P. T. P., Traupmann, K. L., \& Brake, S. Does delay of reinforcement produce durable persistence? Quarterly Journal of Experimental Psychology, 1974, 26, 218-228.

\section{NOTES}

1. The McCuller et al. report focused on the effect of differential FR training on CRF runway acquisition and extinction.

2. The differential treatment preceded the FR 10 reacquisition and extinction reported in this section by a total of 44 days of CRF runway acquisition and extinction, plus the 2-month vacation.

(Received for publication June 15, 1976; accepted August 13, 1976.) 